\title{
Dermatite alérgica à picada de insetos em ovinos no estado do Pará1
}

\author{
José Diomedes Barbosa2*, Tatiane Teles Albernaz², Carlos Magno Chaves \\ Oliveira $^{2}$, Marcos Dutra Duarte ${ }^{2}$, Cairo Henrique Sousa de Oliveira ${ }^{3}$, Marilene \\ de Farias Brito ${ }^{4}$ e André Guimarães Maciel e Silva ${ }^{2}$
}

\begin{abstract}
Barbosa J.D., Albernaz T.T., Oliveira C.M.C., Duarte M.D., Oliveira C.H.S., Brito M.F. \& Silva A.G.M. 2011. [Allergic dermatitis to insect bites in sheep in the state of Pará.] Dermatite alérgica à picada de insetos em ovinos no estado do Pará. Pesquisa Veterinária Brasileira 31(2):117-120. Central de Diagnóstico Veterinário, Universidade Federal do Pará, Rua Maximino Porpino da Silva 1000, Pirapora, Castanhal, PA 68743-080, Brazil. E-mail: diomedes@ufpa.br

A skin disease was studied in 159 sheep on 15 farms located in seven counties of northeastern and southeastern of the state of Pará and in one farm in the state of Roraima, Brazil. The disease affected mainly two to four year-old sheep of the Santa Ines breed and crossbreds. Clinical signs were characterized by alopecia, multifocal erythema, small papules, and crusts in several areas of the body, mainly around the eyes. Severe itching in the affected areas, restless, weight loss and lacrimation were also observed. Insects were always seen around the animals. Insects apparently associated with the lesions were captured and identified as pertaining to the genera Simulium and Hippelates. Biopsies of the skin lesions of 10 sheep revealed inflammatory non suppurative mononuclear infiltrates of the dermis with presence of eosinophils. The epidemiologic, clinical and pathological studies of the cases allowed the diagnosis of allergic dermatitis due to the insect bites.
\end{abstract}

INDEX TERMS: Skin lesions, allergic dermatitis, insect bites, Simulium, Hippelates, sheep.

RESUMO.- Foi estudada uma doença em 159 ovinos em 15 propriedades localizadas em sete municípios das mesorregiões nordeste e sudeste do estado do Pará e uma em um município do estado de Roraima. Os ovinos da raça Santa Inês e seus mestiços, de ambos os sexos e com dois a quatro anos de idade, eram os mais acometidos. A doença caracterizava-se por alopecia em diversas regiões do corpo, principalmente ao redor dos olhos; essas lesões evoluíam para eritemas multifocais, pequenas pápulas e crostas. Os animais apresentavam prurido in-

\footnotetext{
${ }^{1}$ Recebido em 2 de agosto de 2010.

Aceito para publicação em 13 de setembro de 2010.

2 Central de Diagnóstico Veterinário (Cedivet), Universidade Federal do Pará (UFPA), Campus Castanhal, Rua Maximino Porpino da Silva 1000, Pirapora, Castanhal, PA 68743-080, Brasil. "Autor para correspondência: diomedes@ufpa.br

${ }^{3}$ Mestrando do Curso de Pós-Graduação em Ciência Animal, Universidade Federal de Minas Gerais (UFMG), Belo Horizonte, MG 30123-970, Brasil.

${ }^{4}$ Departamento de Epidemiologia e Saúde Pública, Instituto de Veterinária, Universidade Federal Rural do Rio de Janeiro (UFRRJ), Seropédica, RJ 23890-000, Brasil. E-mail: marilene@ufrrj.br
}

tenso no local das lesões, inquietação, perda de peso e corrimento ocular. Sempre era observada a presença de mosquitos ao redor dos animais. Com o auxílio de um puçá foi realizada a captura de insetos associados às lesões, durante o repasto nos ovinos, para a identificação. Foram identificados insetos dos gêneros Simulium e Hippelates. Realizou-se biópsia da pele lesada de 10 ovinos. As lesões histológicas se caracterizaram por leves infiltrados inflamatórios mononucleares na derme, com presença de eosinófilos. Os estudos epidemiológicos, clínicos e patológicos desses casos, bem como o descarte dos diagnósticos diferenciais, permitiram concluir que se trata de dermatite alérgica por picada de insetos.

TERMOS DE INDEXAÇÃO: Lesões de pele, ovinos, dermatite alérgica, picada de insetos, Simulium, Hippelates,

\section{INTRODUÇÃO}

A dermatite alérgica por picada de insetos é uma enfermidade que ocorre em locais de clima temperado e subtropical; já foi diagnosticada em ovinos, caprinos, equinos, bovinos, caninos, felinos e humanos, em várias regiões 
do mundo, inclusive Israel, Colômbia, Austrália e Brasil (Mason \& Evans 1991, Yeruham et al. 2000, Corrêa 2005).

Esta enfermidade, também denominada de dermatite de verão, eczema e hipersensibilidade a Culicoides, dermatite estival recidivante crônica, dermatite alérgica sazonal e dermatite atópica, caracteriza-se por uma dermatite crônica com prurido, lesões ulcerativas, pápulas, formação de crostas eritematosas e alopecia. As lesões ocorrem principalmente nas orelhas, ao redor dos olhos e na região ventral do abdômen e acometem animais de diversas idades e raças (Yeruham et al. 2000, Ferreira 2001, Corrêa et al. 2005).

No Brasil a dermatite alérgica foi descrita no Rio Grande do Sul em ovinos de várias raças, com prevalência variável, podendo chegar a $80 \%$ e também em equinos (Schild et al. 2003, Ferreira 2007, Rissi et al. 2010); na Paraíba, a enfermidade foi diagnosticada em um ovino e os sinais observados foram prurido, áreas de alopecia e espessamento da pele, localizados nas orelhas, focinho, pescoço, flanco esquerdo, cauda e testículos (Macêdo et al. 2008).

No Brasil essa enfermidade tem sido associada a mosquitos do gênero Culicoides (Corrêa et al. 2005, Ferreira 2007). Em outros países algumas espécies deste gênero têm sido responsabilizadas pelas lesões, e em Israel, além do mosquito, também foi observada a presença de pulgas da espécie Ctenocephalides felis (Yeruham et al. 2004).

A mortalidade é baixa, porém as perdas econômicas são consideráveis, uma vez que as lesões disseminamse pelo corpo do animal, causam danos à lã e determinam seu desprendimento. Observa-se acentuada perda de peso nos ovinos doentes e alguns podem morrer em consequência de infecções secundárias graves (Corrêa et al. 2005).

\section{MATERIAL E MÉTODOS}

Dados epidemiológicos e clínico-patológicos foram obtidos através de visitas clínicas realizadas a 15 propriedades, localizadas em sete municípios das mesorregiões nordeste e sudeste do estado do Pará e uma em um município do estado de Roraima.

Foram examinados 159 ovinos. A partir do exame clínico, catalogaram-se as observações relativas à localização, característica e intensidade das lesões.

A captura de insetos associados às lesões foi realizada, durante o repasto nos ovinos, com o auxílio de um puçá. Os insetos foram colocados em frascos de vidro contendo álcool a $70 \%$ e enviados ao Laboratório de Parasitologia da Universidade Federal de Minas Gerais para a identificação.

Realizou-se biópsia da pele lesada de 10 ovinos, em três propriedades. O material foi fixado em formol a $10 \%$ e processado para exame histopatológico no Laboratório de Patologia Veterinária do Projeto Sanidade Animal da Universidade Federal Rural do Rio de Janeiro (SAP 29312-29317).

\section{RESULTADOS}

A doença ocorre, geralmente, na época chuvosa, que na região compreende os meses de dezembro a maio, mas pelo fato de não se tratar os animais afetados, alguns permanecem com lesões durante todo o ano. Os ovinos da raça Santa Inês e seus mestiços, de ambos os sexos e com dois a quatro anos de idade, eram os mais acometidos.

Durante o processo de captura dos insetos não foi observada preferência por uma região do corpo do animal; o repasto era realizado em várias regiões do corpo dos ovinos. Quando estavam sendo picados manifestavam inquietação acentuada e apresentavam prurido intenso no local da lesão, perda de peso, corrimento ocular, lesões de pele caracterizadas por alopecia.

Essas lesões se iniciavam como eritema e alopecia, geralmente ocorriam ao redor dos olhos, e se estendiam para outras regiões como face, orelhas, pescoço (Fig.1A,B), cernelha, garupa, membros, base da cauda, vulva, períneo e bolsa escrotal; após evoluíam para eritemas multifocais e formação de pequenas pápulas.

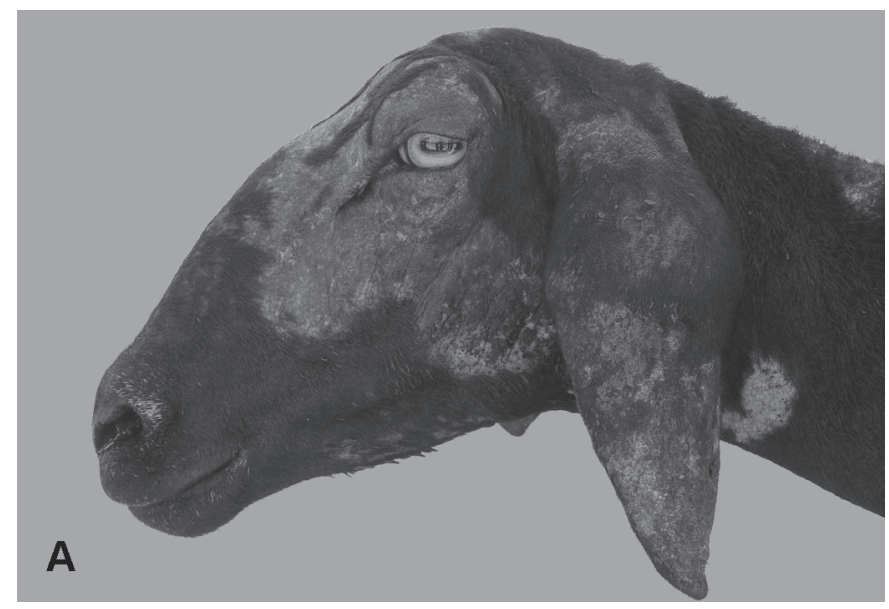

Fig.1.A,B. Ovino Santa Inês, procedente do estado do Pará, com dermatite alérgica à picada de insetos, caracterizada por alopecia e crostas na região da face, orelhas e pescoço.

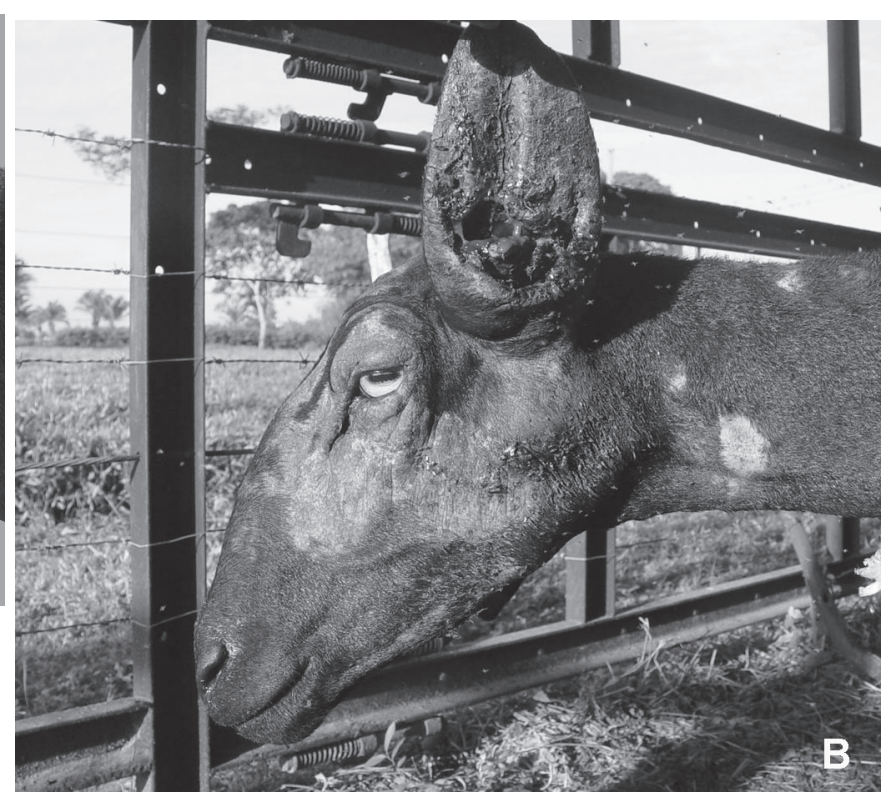


Em alguns casos a pele apresentava-se rugosa, engrossada e edemaciada, com formação de crostas e rachaduras na epiderme, a qual apresentava exsudação serossanguinolenta, com características de lesões crônicas.

Em função do prurido eram comuns escoriações da pele por autotraumatismo; em alguns casos os animais apresentavam otohematoma e perda de parte das orelhas.

Foram identificados insetos dos gêneros Simulium e Hippelates.

Nas biópsias de pele, as lesões histológicas se caracterizaram por leves infiltrados inflamatórios não purulentos (mononucleares) na derme, com presença de eosinófilos.

\section{DISCUSSÃO E CONCLUSÃO}

As lesões macroscópicas e histológicas evidenciadas nos animais deste estudo demonstraram que a doença é uma dermatite alérgica sazonal causada pela picada de insetos, similar à enfermidade descrita em ovinos em outros estados do Brasil (Schild et al. 1993, Corrêa 2005, Macêdo et al. 2008).

As lesões histológicas caracterizadas por dermatite com infiltrado de eosinófilos ocorrem em casos de hipersensibilidade imediata (Connan \& Lloyd 1988, Schild et al. 1993, Ferreira 2001, Souza et al. 2005). Essa reação alérgica provavelmente ocorre em resposta às proteínas presentes na saliva dos insetos; em indivíduos sensíveis, há produção de anticorpos lgE, que reagem especificamente com o alérgeno. Segundo Holmes (1991), a resposta inflamatória e o prurido intenso ocorrem como consequência da degranulação de mastócitos presentes na pele, os quais liberam mediadores químicos como a histamina.

A dermatite com infiltrado inflamatório de células mononucleares tem sido observada nos casos crônicos da doença e deve-se provavelmente ao autotraumatismo induzido pelo prurido e às infecções secundárias que frequentemente ocorrem nos animais afetados, como também foi descrito por Corrêa (2005).

Segundo Baba \& Takaoka (1991), os insetos do gênero Simulium são conhecidos como "borrachudos" ou "piuns"; são insetos holometábolos, de tamanho relativamente pequeno, de cor geralmente frisado a negro ou com tons castanhos amarelados. Apenas as fêmeas adultas são hematófagas, os demais se alimentam de seiva vegetal. De acordo com Coscaron (1981), o ato da picada é rápido e silencioso, e dura de 3-8 minutos. No princípio as picadas são indolores devido às propriedade anestésicas da saliva (Ramirez-Perez 1977).

Os insetos do gênero Hippelates conhecidos como "moscas-dos-olhos", "lambe-olhos", "mosca-cachorro" e "mosquito-remela" são moscas bem pequenas, que possuem o hábito de lamber o canto dos olhos do homem ou dos animais e às vezes causam ulcerações e infecções (Wikipédia 2010).

Possivelmente a associação desses insetos seja responsável pela patogenia da enfermidade, de forma que a picada inicial dos mosquitos hematófagos do gênero Simulium, provoca pequenas exsudações na pele que atraem as moscas lambedoras do gênero Hippelates e desen- cadeiam o processo alergênico, que leva ao prurido intenso e ao ato dos animais se coçarem e traumatizarem diferentes regiões do corpo.

No Rio Grande do Sul, a dermatite alérgica está associada à picada de insetos dos gêneros Anopheles e Culicoides (Corrêa 2005); nos rebanhos de ovinos criados nas fazendas localizadas no bioma amazônico não foram identificados insetos desses gêneros.

O diagnóstico diferencial deve ser feito com outras enfermidades que causam lesões semelhantes na pele de ovinos, tais como scrapie, sarna psoróptica e fotossensibilização hepatógena, que também causam em ovinos intenso prurido e perda de lã (Driemeier 2001, Berne \& Farias 2001, Albernaz et al. 2010). Entretanto, scrapie ocorre principalmente em animais maiores de 42 meses e manifestase em poucos animais em um rebanho com sinais clínicos neurológicos, acompanhados de prurido. Em ovinos com sarna é possível encontrar-se o ácaro em raspados de pele e pelos, e as lesões são observadas em áreas cobertas por Iã. A fotossensibilização hepatógena causada por ingestão de Brachiaria brizantha também causa dermatite, porém as lesões de pele são associadas à lesão hepática.

Conclui-se que a dermatite alérgica está associada aos insetos dos gêneros Simulium e Hippelates nos rebanhos estudados e está muito difundida pelas mesorregiões nordeste e sudeste do estado do Pará, durante os meses de dezembro a maio, por ser o período mais chuvoso, no qual se observa maior proliferação de insetos.

\section{REFERÊNCIAS}

Albernaz T.T. 2010. Fotossensibilização em ovinos associada à ingestão de Brachiaria brizantha no estado do Pará. Dissertação de Mestrado em Ciência Animal, Universidade Federal do Pará. 50p.

Baba M. \& Takaoka H. 1991. Oviposition habitats of a univoltine blackfly, Prosimulium Kiotoense (Diptera: Simuliidae), in Kyushu, Japan. Med. Vet. Entomol. 5:351-357.

Berne M.E.A. \& Farias N.A. 2001. Sarna, p.52-59. In: Riet-Correa F., Schild A.L., Méndez M.C. \& Lemos R.A.A. (Eds), Doenças dos Ruminantes e Equinos. Vol.2. Editora Varela, São Paulo.

Connan R.M. \& Lloyd S. 1988. Seasonal allergic dermatitis in sheep. Vet. Rec. 124:335-337.

Corrêa T.G. 2005. Etiologia e epidemiologia da dermatite alérgica sazonal em ovinos no sul do Rio Grande do Sul. Dissertação de Mestrado em Parasitologia, Departamento de Microbiologia e Parasitologia, Instituto de Biologia, Universidade Federal de Pelotas. 47p.

Coscaron S. 1981. Insecta, Diptera, Simuliidae. Fauna Agua Dulce de la República Argentina 38(1):1-105.

Driemeier D. 2001. Scrapie, p.391-396. In: Riet-Correa F., Schild A.L., Méndez M.C. \& Lemos R.A.A. (Eds), Doenças dos Ruminantes e Equinos. Editora Varela, São Paulo.

Ferreira J.L.M. 2001. Dermatite alérgica sazonal, p.505-507. In: RietCorrea F., Schild A.L., Méndez M.C. \& Lemos R.A.A. (Eds), Doenças dos Ruminantes e Equinos. Vol.2. Editora Varela, São Paulo.

Ferreira J.L.M. 2007. Dermatite alérgica sazonal, p.624-626. In: RietCorrea F., Schild A.L., Lemos R.A.A. \& Borges J.R.J. (Eds), Doen-

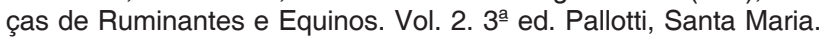

Holmes M.A. 1991. The epidemiology and possible MHC linkage of Culicoides hypersensitivity. Equine Vet. J. 23(4):239-240.

Macêdo J.T.S.A., Riet-Correa F., Dantas A.F.M. \& Simões S.V.D. 2008. 
Doenças da pele em ovinos e caprinos no semi-árido brasileiro. Pesq. Vet. Bras. 28(12):633-642.

Mason K.V. \& Evans A.G. 1991. Mosquito bite-caused eosinophilic dermatitis in cats. J. Am. Vet. Med. Assoc. 198(12):2086-2088.

Ramires-Perez J. 1977. Estudio sobre la morfologia de Simulium metalicum: vector de la encorcercosis humanas em Venezuela. Publ. Cient. 388, Organizacion Panamericana de la Salud, Washington, DC. 140p.

Rissi D.R., Pierezan F., Oliveira Filho J.C., Fighera R.A., Irigoyen L.F., Kommers G.D. \& Barros C.S.L. 2010. Doenças de ovinos da região Central do Rio Grande do Sul: 361 casos. Pesq. Vet. Bras. 30(1):2128.

Schild A.L., Ferreira J.L. \& Soares M.P. 2003. Boletim no.23 do Laboratório Regional de Diagnóstico. Editora e Gráfica Universitária UFPEL, Pelotas RS, p.40.
Schild A.L., Riet-Correa F., Ferreira J.L. \& Méndez M.C. 1993. Boletim no.13 do Laboratório Regional de Diagnóstico. Editora e Gráfica Universitária UFPEL, Pelotas RS, p.45.

Souza T.M., Fighera R.A., Piazer J.V., Irigoyen L.F. \& Barros C.S.L. 2005. Dermatite alérgica sazonal em ovinos. Ciência Rural 35(2):475477.

Yeruham I., Braverman Y. \& Perl S. 2000. Study of apparent hypersensitivity to Culicoides species in sheep in Israel. Vet. Rec. 147:360363.

Yeruham I., Perl S. \& Braverman Y. 2004. Seasonal allergic dermatitis in sheep associated with Ctenocephalides and Culicoides bites. Vet. Dermatol. 15:377-380.

Wikipédia. 2010. Disponível em <http://pt.wikipedia.org/wiki/Mosca-dosolhos> Acesso 12 ago. 2010. 